J. Nonlinear Var. Anal. 4 (2020), No. 1, pp. 5-20

Available online at http://jnva.biemdas.com

https://doi.org/10.23952/jnva.4.2020.1.02

\title{
SOME TOPICS IN VECTOR OPTIMIZATION VIA IMAGE SPACE ANALYSIS
}

\author{
G. MASTROENI ${ }^{1, *}$, M. PAPPALARDO ${ }^{1}$, F. RACITI $^{2}$ \\ ${ }^{1}$ Department of Computer Science, University of Pisa, L.go B. Pontecorvo, 356127 Pisa, Italy \\ ${ }^{2}$ Department of Mathematics and Computer Science, University of Catania, V.le A. Doria, 695125 Catania, Italy
}

\begin{abstract}
In this paper, we outline the main features of the image space analysis applied to a vector optimization problem. By means of separation arguments in the image space associated with a vector optimization problem, several theoretical aspects can be developed: in particular, we focus our attention on saddle point and first order optimality conditions, regularity and constraint qualifications.
\end{abstract}

Keywords. Image space; Separation; Vector optimization.

\section{INTRODUCTION}

The image space analysis has shown to be a powerful tool and a unifying scheme for studying both Vector Optimization and Vector Variational Inequalities [9, 13]. Giannessi formally introduced this approach showing, more generally, that it can be applied to any kind of problem $(P)$ that can be expressed under the form of the impossibility of a parametric system [6, 7, 14].

The impossibility of such a system is reduced to the disjunction of two suitable subsets $\mathscr{K}$ and $\mathscr{H}$ of the image space: $\mathscr{K}$ is defined by the image of the functions involved in $(P)$, while $\mathscr{H}$ is a convex cone that depends on the type of constraints which define $(P)$. The disjunction between $\mathscr{K}$ and $\mathscr{H}$ was proved by showing that they lie in two disjoint level sets of a separating functional. If such a functional can be found linear, then we say that $\mathscr{K}$ and $\mathscr{H}$ admit a linear separation. A suitable subclass of separating functionals is said to be regular iff the separation obtained by means of a functional of such a subclass guarantees the disjunction between $\mathscr{K}$ and $\mathscr{H}$. Exploiting separation arguments in the image space, several theoretical aspects can be developed, such as, duality, Lagrangian-type optimality conditions, regularity, and penalization. Duality arises from the existence of a linear separation between $\mathscr{K}$ and $\mathscr{H}$, while necessary optimality conditions arise from the linear separation between a convex conical approximation of $\mathscr{K}$ and $\mathscr{H}$ [8]. Penalization turns out to be closely related to nonlinear separation [6, 16]. The existence of a regular separating functional characterizes the regularity of the considered problem in view of the definition of the Lagrangian-type optimality conditions. Let us remark that the concepts presented in this article have been applied in the modeling of various equilibrium problems, see, e.g., $[18,19]$ for a detailed analysis of the vector traffic equilibrium problem.

\footnotetext{
${ }^{*}$ Corresponding author.

E-mail addresses: giandomenico.mastroeni@unipi.it (G. Mastroeni), massimo.pappalardo@unipi.it (M. Pappalardo), fraciti@dmi.unict.it (F. Raciti).

Received October 30, 2019; Accepted March 9, 2020.
}

(C)2020 Journal of Nonlinear and Variational Analysis 
The paper is organized as follows. In Section 2, we introduce the general features of the image space analysis applied to a vector optimization problem (VOP). In Section 3, we analyze the main existence results for a VOP and their relations with suitable properties of the image associated with VOP. Sections 4 and 5 are devoted to the analysis of linear separation in the image space and its regularity. In Sections 6 and 7, sufficient and necessary optimality conditions for VOP are presented by making use of the separation techniques developed in the image space. In particular, in Section 7, we outline the concept of $G$-semidifferentiability of a function, introduced by Giannessi [8], in order to treat in an axiomatic way the theory of generalized directional derivatives that were used in the definition of necessary optimality conditions.

Let us introduce the main notations and definitions that will be used throughout the paper. Let $V$ be a real topological linear space, and $\mathbf{R}_{+}^{n}:=\left\{x \in \mathbf{R}^{n}: x \geq 0\right\}$. A set $\mathscr{H} \subseteq V$ is said to be a cone iff $\lambda \mathscr{H} \subseteq \mathscr{H}$, with $\lambda>0$, and a convex cone iff, in addition, $\mathscr{H}+\mathscr{H} \subseteq \mathscr{H}$, where $\mathscr{H}+\mathscr{H}=\left\{h_{1}+h_{2} \in V: h_{1} \in \mathscr{H}, h_{2} \in \mathscr{H}\right\} ;$ cone $\mathscr{H}=\{y \in V: y=\lambda x, \quad \lambda>0, \quad x \in \mathscr{H}\}$. The closure, the interior, the relative interior, the relative boundary and the convex hull of a set $\mathscr{H}$ are denoted by $c l \mathscr{H}$, int $\mathscr{H}$, ri $\mathscr{H}$, rbd $\mathscr{H}$, and conv $\mathscr{H}$, respectively. aff $(\mathscr{H})$ and lin $(\mathscr{H})$ will denote the smallest affine variety and the smallest subspace that contains $\mathscr{H}$, respectively. $\pi\left(\mathbf{R}^{\ell} ; K\right)$ will denote the projection of the set $K \subseteq \mathbf{R}^{\ell} \times \mathbf{R}^{m}$ on $\mathbf{R}^{\ell}$.

$$
\mathscr{H}^{*}=\left\{y \in V^{*}:\langle x, y\rangle \geq 0, \quad \forall x \in \mathscr{H}\right\}
$$

is the positive polar of $\mathscr{H}$, where $\langle\cdot, \cdot\rangle$ is the duality pairing between $V$ and its topological dual $V^{*}$.

Given a cone $D \subset \mathbf{R}^{n} a, b \in \mathbf{R}^{n}$, the inequality $a \nsupseteq{ }_{D} b$ means $a-b \notin D$. A closed and convex cone $C \subset V$ is called pointed iff $C \cap(-C)=\{0\}$. Let $\mathscr{X}$ be a convex subset of a linear space and $f: \mathscr{X} \longrightarrow V . f$ is called $C$-convex on $\mathscr{X}$ iff $\forall x_{1}, x_{2} \in \mathscr{X}$,

$$
(1-\alpha) f\left(x_{1}\right)+\alpha f\left(x_{2}\right)-f\left((1-\alpha) x_{1}+\alpha x_{2}\right) \in C, \quad \forall \alpha \in(0,1) .
$$

\section{Image of A Vector Optimization Problem}

Let $X$ be a Banach space and consider the following vector optimization problem,

$$
\min _{C} f(x) \quad \text { s.t. } \quad x \in R:=\{x \in X: g(x) \in D\},
$$

where $f: X \rightarrow \mathbf{R}^{\ell}, g: X \rightarrow \mathbf{R}^{m}, C$ is a pointed cone in $\mathbf{R}^{\ell}, D$ is a closed convex cone in $\mathbf{R}^{m}$. $y \in R$ is a vector minimum point (for short, v.m.p.) of (P) iff

$$
f(y) \Varangle_{C \backslash\{0\}} f(x), \quad \forall x \in R .
$$

When $C=\mathbf{R}_{+}^{\ell}$, (P) becomes the classic Pareto vector problem. $y \in R$ is a weak v.m.p. of (P) iff

$$
f(y) \gtrless_{\text {int } C} f(x), \quad \forall x \in R,
$$

When $C=\mathbf{R}_{+}^{\ell}$, (P) becomes the classic weak Pareto vector problem.

It is trivial to note that (2.1) is satisfied iff the system (in the unknown $x$ ):

$$
f(y)-f(x) \geq_{C \backslash\{0\}} 0, g(x) \geq_{D} 0, x \in X
$$

is impossible. Consider the sets

$$
\begin{aligned}
& \mathscr{K}_{y}:=\left\{(u, v) \in \mathbf{R}^{\ell} \times \mathbf{R}^{m}: u=f(y)-f(x), v=g(x), x \in X\right\} . \\
& \mathscr{H}:=\left\{(u, v) \in \mathbf{R}^{\ell} \times \mathbf{R}^{m}: u \geq_{C \backslash\{0\}} 0, v \geq_{D} 0\right\}=(C \backslash\{0\}) \times D
\end{aligned}
$$


and

$$
\mathscr{H}_{u}:=\left\{(u, v) \in \mathbf{R}^{\ell} \times \mathbf{R}^{m}: u \geq_{C \backslash\{0\}} 0, v=0\right\}=(C \backslash\{0\}) \times\{0\}
$$

$\mathscr{K}_{y}$ is called the image associated with $(\mathrm{P})$.

We observe that the impossibility of the system (2.3) is equivalent to the condition

$$
\mathscr{K}_{y} \cap \mathscr{H}=\emptyset .
$$

So, $y \in R$ is a v.m.p. iff (2.4) holds.

Similarly, if we put $\mathscr{H}=\operatorname{int} C \times D$, then $y$ is a weak v.m.p. for (P) iff (2.4) holds.

To directly prove whether $\mathscr{K}_{y} \cap \mathscr{H}=\emptyset$ or not, is generally too difficult. Therefore, in order to show such a disjunction, it will be proved that the two sets lie in two disjoint level sets of a functional.

In general, $\mathscr{K}_{y}$ is not convex even when the functions involved enjoy some convexity properties. To overcome this difficulty, we introduce a regularization of the image $\mathscr{K}_{y}$, namely, the conic extension with respect to $\mathrm{cl} \mathscr{H}$, denoted by $\mathscr{E}_{y}$ :

$$
\mathscr{E}_{y}:=\left\{(u, v) \in \mathbf{R}^{\ell} \times \mathbf{R}^{m}: u \leq_{C} f(y)-f(x), v \leq_{D} g(x), x \in X\right\}=\mathscr{K}_{y}-c l \mathscr{H} .
$$

From now on, when there is no confusion, we will avoid mentioning the dependence of the sets $\mathscr{K}_{y}$ and $\mathscr{E}_{y}$ on $y \in R$.

The importance of the conic extension is enforced by the following statement.

Proposition 2.1. Condition (2.4) holds iff

$$
\mathscr{E} \cap \mathscr{H}=\emptyset
$$

or

$$
\mathscr{E} \cap \mathscr{H}_{u}=\emptyset .
$$

Proof. The equivalence between (2.4) and (2.5) follows from

$$
\mathscr{H}+c l \mathscr{H}=\mathscr{H} .
$$

We show that (2.5) and (2.6) are equivalent.

Obviously, (2.5) implies (2.6) since $\mathscr{H}_{u} \subseteq \mathscr{H}$. To prove the reverse implication, we suppose that (2.6) holds and there exists $(\bar{u}, \bar{v}) \in \mathscr{E} \cap \mathscr{H}$. Since

$$
\mathscr{E}-c l \mathscr{H}=\mathscr{K}-(\operatorname{cl} \mathscr{H}+\operatorname{cl} \mathscr{H})=\mathscr{E},
$$

we have $(\bar{u}, \bar{v})-(0, \bar{v})=(\bar{u}, 0) \in \mathscr{E}$. Since $(\bar{u}, 0) \in \mathscr{H}_{u}$, this leads to a contradiction. This completes the proof.

Proposition 2.2. If we let $\mathscr{H}=(C \backslash\{0\}) \times D$, (resp. $\mathscr{H}=$ int $C \times D)$, then y is a v.m.p. (resp. weak v.m.p.) for $(P)$ iff

$$
\mathscr{E} \cap \mathscr{H}=\emptyset .
$$

Exploiting the fact that $\mathscr{H}$ is a cone, we obtain a further characterization of the optimality condition in the image space.

Corollary 2.1. (2.8) is equivalent to

$$
\text { cone } \mathscr{E} \cap \mathscr{H}=\emptyset .
$$




\section{EXISTENCE OF A VECTOR MINIMUM POINT}

The existence of a v.m.p. of problem (P) is closely related to the properties of the image $f(R)$ of the feasible set. The most important ones are those of cone compactness $[11,21]$ that we will briefly recall.

Definition 3.1. Let $C$ be a cone in $\mathbf{R}^{\ell}$. A set $Y \subset \mathbf{R}^{\ell}$ is said to be $C$-compact, iff, for any $y \in Y$, the set $(y-c l C) \cap Y$ is compact.

As shown in the Remark 3.2.2 of [21] a $C$-compact set is not necessarily compact. Consider, for example, the set $Y=\left\{y \in \mathbf{R}^{2}: y_{1}+y_{2} \geq 0\right\}$ and $C=\mathbf{R}_{+}^{2}$.

The following theorem is a first important existence result for $(\mathrm{P})$. We make the blanket assumption that $f(R)$ is nonempty.

Theorem 3.1. If $f(R)$ is C-compact, then admits a v.m.p..

Proof. Taking into account Proposition 3.2.1 of [21], we can obtain from Theorem 3.3 the desired conclusion immediately.

Since the conic extension $\mathscr{E}$ involves the image of both feasible and unfeasible points, in order to investigate the existence of the v.m.p. of (P), we consider the following subset of $\mathscr{E}$

$$
\mathscr{E}_{u}:=\mathscr{E} \cap\{(u, v) \in C \times\{0\}\} .
$$

\section{Proposition 3.1.}

$$
\mathscr{H} \cap \mathscr{E}=\emptyset \Longleftrightarrow \mathscr{H} \cap \mathscr{E}_{u}=\emptyset
$$

Proof. Since $\mathscr{E}_{u} \subseteq \mathscr{E}$, we only have to prove that

$$
\mathscr{H} \cap \mathscr{E}_{u}=\emptyset \Longrightarrow \mathscr{H} \cap \mathscr{E}=\emptyset .
$$

Suppose that $\mathscr{H} \cap \mathscr{E} \neq \emptyset$. Then there exists $\left(u^{*}, v^{*}\right) \in \mathscr{K}$ such that $u^{*}-h_{1} \geq_{C \backslash\{0\}} 0, v^{*}-h_{2} \geq$ $0, h_{1} \in c l C, h_{2} \geq 0$. Therefore $v^{*} \geq h_{2} \geq 0$ and the point

$$
\left(u^{*}, v^{*}\right)-\left(h_{1}, v^{*}\right)=\left(u^{*}-h_{1}, 0\right) \in \mathscr{E}_{u} \cap \mathscr{H},
$$

which contradicts the hypothesis. This completes the proof.

Proposition 3.2. Let $f(R)$ be closed. If $\mathscr{E}_{u}$ is a bounded set, then $(P)$ admits a v.m.p..

Proof. Observe that

$\mathscr{E}_{u}=\left\{(u, v) \in \mathbf{R}^{\ell} \times \mathbf{R}^{m}: u=f(y)-f(x)-h_{1}, v=g(x)-h_{2}, x \in X, h_{1} \in c l C, h_{2} \geq 0\right\} \cap(C \times\{0\})$.

We have

$$
\begin{aligned}
\pi\left(\mathbf{R}^{\ell} ; \mathscr{E}_{u}\right)=\left\{u \in \mathbf{R}^{\ell}: u\right. & \left.=f(y)-f(x)-h_{1}, x \in K, h_{1} \in C\right\} \cap C \\
& =[f(y)-f(R)-C] \cap C \supseteq[f(y)-f(R)] \cap C .
\end{aligned}
$$

Since $\mathscr{E}_{u}$ is a bounded set, we have that $\pi\left(\mathbf{R}^{\ell} ; \mathscr{E}_{u}\right)$ is a bounded set. Therefore $[f(y)-f(R)] \cap C$ is compact since it is a closed subset of $\pi\left(\mathbf{R}^{\ell} ; \mathscr{E}_{u}\right)$.

It is easy to show that the set $[f(y)-C] \cap f(R)$ is compact. Indeed, it is obtained by an affine transformation of the set $[f(y)-f(R)] \cap C$. Therefore the set $f(R)$ is $C$-compact. From Theorem 3.1, we complete the proof immediately. 
In Proposition , $f(R)$ is assumed to be closed. This condition, together with the boundedness of $\mathscr{E}_{u}$, ensures the $C$-compactness of $f(R)$. In fact, the conic extension $\mathscr{E}$ provides a regularization of $f(R)$ that no longer ensures the closedness of $f(R)$ even though $\mathscr{E}$ turns out to be closed. To overcome this difficulty, we can define a further extension of the set $\mathscr{K}$ that does not involve the objective functions, namely,

$$
\mathscr{E}^{\prime}:=\left[\mathscr{K}-\left(0 \times \mathbf{R}_{+}^{m}\right)\right]
$$

and the corresponding

$$
\mathscr{E}_{v}:=\mathscr{E}^{\prime} \cap\{(u, v) \in C \times\{0\}\} .
$$

Another possibility is to consider the image $\mathscr{K}$ and the subset of the feasible images

$$
\mathscr{K}_{v}:=\mathscr{K} \cap\left\{(u, v) \in C \times \mathbf{R}_{+}^{m}\right\} .
$$

We observe that, in both sets $\mathscr{E}_{v}$ and $\mathscr{K}_{v}$, the objective functions have not been altered, so that it is possible to consider the closedness assumptions directly on the above sets. Adapting again the proof of the Proposition 3.2, we can obtain the following result [15].

Proposition 3.3. If one of the following conditions holds:

(i) $\mathscr{E}_{v}$ is a compact set;

(ii) $\mathscr{K}_{v}$ is a compact set,

then $(P)$ admits a v.m.p..

Proof. (i) We have

$$
\mathscr{E}_{v}:=\left\{(u, v) \in \mathbf{R}^{\ell} \times \mathbf{R}^{m}: u=f(y)-f(x), v=g(x)-h, x \in X, h \geq 0\right\} \cap(C \times\{0\}) .
$$

It follows that

$$
\pi\left(\mathbf{R}^{\ell} ; \mathscr{E}_{v}\right)=\left\{u \in \mathbf{R}^{\ell}: u=f(y)-f(x), x \in R,\right\} \cap C=[f(y)-f(R)] \cap C .
$$

Since $\mathscr{E}_{v}$ is a compact set, we have that $\pi\left(\mathbf{R}^{\ell} ; \mathscr{E}_{v}\right)$ is a compact set. With the same arguments used in the proof of the Proposition 3.2, we have that $[f(y)-C] \cap f(R)$ is compact so that $f(R)$ is $C$-compact. By Theorem 3.1, we complete the proof of part (i).

(ii) We have

$$
\mathscr{K}_{v}:=\left\{(u, v) \in \mathbf{R}^{\ell} \times \mathbf{R}^{m}: u=f(y)-f(x), v=g(x), x \in X\right\} \cap\left(C \times \mathbf{R}_{+}^{m}\right\} .
$$

It follows that

$$
\pi\left(\mathbf{R}^{\ell} ; \mathscr{K}_{v}\right)=\left\{u \in \mathbf{R}^{\ell}: u=f(y)-f(x), x \in R,\right\} \cap C=[f(y)-f(R)] \cap C .
$$

Since $\mathscr{K}_{v}$ is a compact set, we have that $\pi\left(\mathbf{R}^{\ell} ; \mathscr{K}_{v}\right)$ is a compact set. With the same arguments used in the proof of part (i), we complete the proof immediately.

The previous existence results were essentially obtained by proving that set $f(R)$ is $C$-compact. It is, therefore, of interest to consider conditions on the objective function $f$ and on the constraint function $g$ that guarantee the $C$-compactness of $f(R)$. A first obvious remark is that this condition holds when $R$ is a compact set and $f$ is a continuous function on $R$. In order to weaken the previous assumptions, it is necessary to deepen the analysis of the notion of $C$-compactness of a set. This has led to consider a slightly weaker property, the $C$-semicompactness [4, 23, 24] and to express the $C$-compactness of a set $Y$ in terms of the $C$-boundedness and $C$-closedness of $Y$, generalizing, in this way, the classic topological results. 
Definition 3.2. $Y \subset \mathbf{R}^{\ell}$ is said to be

(i) $C$-closed iff $Y+c l C$ is closed;

(ii) $C$-bounded iff $Y^{+} \cap(-c l C)=\{0\}$, where $Y^{+}:=\left\{y^{\prime} \in \mathbf{R}^{\ell}\right.$ : there exist sequences $\left\{\alpha_{k}\right\} \subset$ $\left(\mathbf{R}_{+} \backslash\{0\}\right)$ and $\left\{y_{k}\right\} \subset Y$ s.t. $\alpha_{k} \rightarrow 0$ and $\left.\alpha_{k} y_{k} \rightarrow y^{\prime}\right\}$.

Definition 3.3. Let $C$ be a cone and $Y$ be a set in $\mathbf{R}^{\ell}$. $Y$ is said to be $C$-semicompact iff every open cover of $Y$ of the form

$$
\left\{\left(y^{\gamma}-c l C\right)^{c}: y^{\gamma}, \gamma \in I\right\}
$$

has a finite subcover, where $I$ is some index set and the superscript $c$ denotes the complement of a set.

The following theorem states the equivalence among the concepts of $C$-compactness, $C$ semicompactness and the ones of $D$-closedness and boundedness under the hypotheses that cl $C$ is a pointed and convex cone and $Y$ is a closed and convex set. It is shown in Proposition 3.2.1 of [21] that if $Y$ is $C$-compact, then $Y$ is $C$-semicompact. In the hypotheses of closedness and convexity of the set $Y$, the previous concepts are equivalent.

Theorem 3.2. Let $C$ be a pointed and convex cone in $\mathbf{R}^{\ell}$. Let $Y$ be a nonempty, closed and convex set in $\mathbf{R}^{\ell}$. Then, the following statements are equivalent:

(i) $Y$ is C-compact;

(ii) $Y$ is $C$-closed and $C$-bounded;

(iii) $Y$ is $C$-semicompact.

Proof. From Proposition 3.2.3 of [21], we can obtain the desired conclusion immediately.

Theorem 3.3. If $f(R)$ is C-semicompact, then $(P)$ admits a v.m.p..

Proof. By putting $Y=f(R), D=C$ and taking into account that $C$ is pointed (i.e., acute in the sense of [21], being $C$ closed and convex) in the setting of problem (P), we can obtain the result from Theorem 3.2.2 of [21] easily.

By means of the concept of $C$-semicontinuity of a vector function, it is possible to extend the classic Weierstrass theorem.

Definition 3.4. [4] Let $C$ be a cone in $\mathbf{R}^{\ell}$. A function $f: \mathbf{R}^{n} \longrightarrow \mathbf{R}^{\ell}$ is said to be $C$-semicontinuous if

$$
f^{-1}(y-c l C)=\left\{x \in \mathbf{R}^{n}: y-f(x) \in c l C\right\}
$$

is closed, $\forall y \in \mathbf{R}^{\ell}$.

Theorem 3.4. Let $X$ be a compact set in $\mathbf{R}^{\ell}$, $f$ be a $C$-semicontinuous function and $g: X \longrightarrow \mathbf{R}^{m}$ be a $\mathbf{R}_{+}^{m}$-semicontinuous function on $X$. Then the set $f(R)$ is $C$-semicompact.

Proof. It is simple to prove that, under the considered hypotheses, the set $R$ is compact so that the statement follows from Lemma 3.2.6 of [21].

Remark 3.1. It is known that $f: \mathbf{R}^{n} \longrightarrow \mathbf{R}^{\ell}$ is $\mathbf{R}_{+}^{\ell}$-semicontinuous iff, for each $f_{i}, \mathrm{i}=1, \ldots, \ell$ is lower semicontinuous. 
In our preceding results, we only considered classic hypotheses of boundedness and compactness on the image $\mathscr{K}$ and on the extended image $\mathscr{E}$. This is due to the dependence of these sets on the point $y \in R$. If we define the image $\mathscr{K}$ independently of the point $y$, namely,

$$
\tilde{\mathscr{K}}:=\left\{(u, v) \in \mathbf{R}^{\ell} \times \mathbf{R}^{m}: u=f(x), v=g(x), x \in X\right\},
$$

then we can directly assume $C$-compactness on $\tilde{\mathscr{K}}$.

The following result holds [15].

Proposition 3.4. If $\tilde{\mathscr{K}}$ is $\left(C \times \mathbf{R}_{+}^{m}\right)$-compact, then $(P)$ admits a v.m.p..

Proof. The hypotheses imply that $\left(y-\left(C \times \mathbf{R}_{+}^{m}\right)\right) \cap \tilde{\mathscr{K}}$ is compact, $\forall y \in \tilde{\mathscr{K}}$, so is

$$
(f(y)-C) \cap f(R)=\pi\left(\mathbf{R}^{\ell} ; y-\left(C \times \mathbf{R}_{+}^{m}\right) \cap \tilde{\mathscr{K}}\right),
$$

that is, $f(R)$ is $C$-compact. Theorem 3.1 allows to prove our statement immediately.

\section{LINEAR SEPARATION}

Separation methods are one of the main tools which can be used to prove (2.4). The simplest one is linear separation that we briefly recall.

Definition 4.1. The sets $\mathscr{K}$ and $\mathscr{H}$ admit a linear separation iff there exist $\theta^{*} \in C^{*}$ and $\lambda^{*} \in D^{*}$, $\left(\theta^{*}, \lambda^{*}\right) \neq 0$ such that

$$
\left\langle\theta^{*}, u\right\rangle+\left\langle\lambda^{*}, v\right\rangle \leq 0, \quad \forall(u, v) \in \mathscr{K},
$$

or, equivalently,

$$
\sum_{i=1}^{\ell} \theta_{i}^{*}\left[f_{i}(y)-f_{i}(x)\right]+\sum_{j=1}^{m} \lambda_{j}^{*} g_{j}(x) \leq 0, \quad \forall x \in X .
$$

We recall that a linear separation is said to be "proper" if both the sets are not contained in the separating hyperplane.

The following result shows that a linear functional separates $\mathscr{K}$ and $\mathscr{H}$ iff it separates $\mathscr{E}$ and $\mathscr{H}$.

Proposition 4.1. Let $\left(\theta^{*}, \lambda^{*}\right) \in \mathscr{H}^{*} \backslash\{0\}$. Then the following statements are equivalent:

(i) $\left\langle\theta^{*}, u\right\rangle+\left\langle\lambda^{*}, v\right\rangle \leq 0, \quad \forall(u, v) \in \mathscr{K}$.

(ii) $\left\langle\theta^{*}, u\right\rangle+\left\langle\lambda^{*}, v\right\rangle \leq 0, \quad \forall(u, v) \in \mathscr{E}$.

Proof. Suppose that (i) holds. Let $\left(h_{1}, h_{2}\right) \in \mathscr{H}$. Since $\left\langle\theta^{*},-h_{1}\right\rangle+\left\langle\lambda^{*},-h_{2}\right\rangle \leq 0$, we have

$$
\left\langle\theta^{*}, u-h_{1}\right\rangle+\left\langle\lambda^{*}, v-h_{2}\right\rangle \leq 0, \quad \forall(u, v) \in \mathscr{K},
$$

and (ii) holds.

(ii) $\Rightarrow$ (i) is obvious since $\mathscr{K} \subseteq \mathscr{E}$.

The previous result allows us to consider condition (2.8), which can be proved by showing that $\mathscr{E}$ and $\mathscr{H}$ lie in two disjoint level sets of a suitable linear functional.

Theorem 4.1. $\mathscr{K}$ and $\mathscr{H}$ admit a proper linear separation iff

$$
\operatorname{ri}(\operatorname{conv} \mathscr{E}) \cap \operatorname{ri} \mathscr{H}=\emptyset .
$$

We can consider the following reformulation of Theorem 4.1. 
Theorem 4.2. $\mathscr{E}$ and $\mathscr{H}$ admit a proper linear separation iff

$$
0 \notin \operatorname{ri}(\operatorname{conv} \mathscr{E}) \text {. }
$$

Proof. For any pair of convex sets $A$ and $B$ in $\mathbf{R}^{m}$ we have

$$
\operatorname{ri}(A+B)=\operatorname{ri}(A)+r i(B), \quad \operatorname{ri}(A)=\operatorname{ri}[\operatorname{cl}(A)] .
$$

Therefore (4.3) is equivalent to

$$
0 \notin \operatorname{ri}(\operatorname{conv} \mathscr{E})-\operatorname{ri}(\mathscr{H})=\operatorname{ri}(\operatorname{conv} \mathscr{E})-\operatorname{ri}(\operatorname{cl} \mathscr{H})=\operatorname{ri}(\operatorname{conv} \mathscr{E}-\operatorname{cl} \mathscr{H}) .
$$

Since for every convex set $B$ and any $S \subseteq \mathbf{R}^{m}$, it holds $\operatorname{conv}(S+B)=\operatorname{conv}(S)+B$, we have

$$
\operatorname{conv} \mathscr{E}-\operatorname{cl} \mathscr{H}=\operatorname{conv}(\mathscr{E}-\operatorname{cl} \mathscr{H})=\operatorname{conv} \mathscr{E},
$$

which completes the proof.

Let

$$
L(\theta ; \lambda, x):=\sum_{i=1}^{\ell} \theta_{i} f_{i}(x)-\sum_{j=1}^{m} \lambda_{j} g_{j}(x), \quad \theta \in C^{*}, \quad \lambda \in D^{*}
$$

be the Lagrangian function associated with $(\mathrm{P})$.

Proposition 4.2. Suppose that $y \in R$. Then $\mathscr{E}$ and $\mathscr{H}$ admit a linear separation iff there exist $\theta^{*} \in C^{*}$ and $\lambda^{*} \in D^{*},\left(\theta^{*}, \lambda^{*}\right) \neq 0$, such that $\left(\lambda^{*}, y\right)$ is a saddle point for $L\left(\theta^{*} ; \lambda, x\right)$ on $D^{*} \times X$.

Proof. Suppose that $\mathscr{E}$ and $\mathscr{H}$ admit a linear separation. Since $y \in R$, we have $\sum_{j=1}^{m} \lambda_{j}^{*} g_{j}(y) \geq 0$. Computing at $x=y(4.2)$, we have

$$
\sum_{j=1}^{m} \lambda_{j}^{*} g_{j}(y)=0
$$

Therefore (4.2) is equivalent to the inequality

$$
L\left(\theta^{*} ; \lambda^{*}, y\right) \leq L\left(\theta^{*} ; \lambda^{*}, x\right), \quad \forall x \in X .
$$

The inequality

$$
L\left(\theta^{*} ; \lambda^{*}, y\right) \geq L\left(\theta^{*} ; \lambda, y\right), \quad \forall \lambda \in D^{*}
$$

is equivalent to

$$
0 \leq \sum_{j=1}^{m} \lambda_{j} g_{j}(y), \quad \forall \lambda \in D^{*}
$$

which is fulfilled since $g(y) \in D$. Vice versa, we assume that there exist $\theta^{*} \in C^{*}$ and $\lambda^{*} \in D^{*}$, $\left(\theta^{*}, \lambda^{*}\right) \neq 0$ such that $\left(\lambda^{*}, y\right)$ is a saddle point for $L\left(\theta^{*} ; \lambda, x\right)$ on $D^{*} \times X$, i.e.,

$$
\begin{array}{ll}
\sum_{i=1}^{\ell} \theta_{i}^{*} f_{i}(y)-\sum_{j=1}^{m} \lambda_{j} g_{j}(y) \leq \sum_{i=1}^{\ell} \theta_{i}^{*} f_{i}(y)-\sum_{j=1}^{m} \lambda_{j}^{*} g_{j}(y), \quad \forall \lambda \in D^{*} \\
\sum_{i=1}^{\ell} \theta_{i}^{*} f_{i}(y)-\sum_{j=1}^{m} \lambda_{j}^{*} g_{j}(y) \leq \sum_{i=1}^{\ell} \theta_{i}^{*} f_{i}(x)-\sum_{j=1}^{m} \lambda_{j}^{*} g_{j}(x), \quad \forall x \in X .
\end{array}
$$

Computing (4.6) for $\lambda=0$, we obtain $\sum_{j=1}^{m} \lambda_{j}^{*} g_{j}(y) \leq 0$. It follows from $g(y) \in D$ that

$$
\sum_{j=1}^{m} \lambda_{j}^{*} g_{j}(y) \geq 0
$$


So, (4.5) is fulfilled. From (4.5), it follows that (4.7) is equivalent to (4.2).

Linear separation can also be characterized in terms of the polarity. Let us recall the following result.

Theorem 4.3. The bipolar $A^{* *}=\left(A^{*}\right)^{*}$ of a subset $A \subset X$ is given by

$$
A^{* *}=\operatorname{cl} \operatorname{conv}(A \cup\{0\}) \text {. }
$$

Next proposition is a straightforward consequence of the definition of linear separation.

Proposition 4.3. $\mathscr{E}$ and $\mathscr{H}$ admit a linear separation iff

$$
(\text { cone } \mathscr{E})^{*} \neq\{0\} \text {. }
$$

From the previous results, we have the following result.

Theorem 4.4. $\mathscr{E}$ and $\mathscr{H}$ admit a linear separation iff

$$
\text { cl cone conv } \mathscr{E} \neq \mathbf{R}^{\ell} \times \mathbf{R}^{m} .
$$

Proof. Taking into account that $0 \in \mathscr{E}$, Proposition 4.3 and Theorem 4.3, (4.9) is equivalent to

$$
\text { cl conv cone } \mathscr{E} \neq \mathbf{R}^{\ell} \times \mathbf{R}^{m} \text {. }
$$

Recalling that conv cone $\mathscr{E}=$ cone conv $\mathscr{E}$, we obtain (4.10).

\section{REGULARITY OF LINEAR SEPARATION}

Assume that the hyperplane of equation

$$
\left\langle\theta^{*}, u\right\rangle+\left\langle\lambda^{*}, v\right\rangle=0
$$

separates the sets $\mathscr{E}$ and $\mathscr{H}$. The existence of a separating hyperplane does not guarantee that $\mathscr{E} \cap \mathscr{H}=\emptyset$. In order to ensure the disjunction of the two sets, some restrictions on the choice of the multipliers $\left(\theta^{*}, \lambda^{*}\right)$ must be imposed.

Proposition 5.1. Assume that $\mathscr{E}$ and $\mathscr{H}$ are separated by hyperplane (5.1).

(i) If $\theta^{*} \in$ int $C^{*}$, then $\mathscr{E} \cap \mathscr{H}=\emptyset$.

(ii) If $\theta^{*} \neq 0$, then $\mathscr{E} \cap($ int $C \times D)=\emptyset$.

Proof. We recall (see e.g. [2]) that $\mathrm{clC}$ is pointed iff $\operatorname{int} C^{*} \neq \emptyset$ and

$$
\operatorname{int} C^{*}=\left\{x^{*} \in C^{*}:\left\langle x, x^{*}\right\rangle>0, \quad \forall x \in c l C, x \neq 0\right\} .
$$

(i) Taking into account that $\theta^{*} \in \operatorname{int} C^{*}$, we have

$$
\left\langle\theta^{*}, u\right\rangle+\left\langle\lambda^{*}, v\right\rangle>0, \quad \forall(u, v) \in \mathscr{H} .
$$

(ii) Taking into account that $\theta^{*} \in C^{*} \backslash\{0\}$, we have

$$
\left\langle\theta^{*}, u\right\rangle+\left\langle\lambda^{*}, v\right\rangle>0, \quad \forall(u, v) \in(\text { int } C \times D) .
$$

The previous inequalities guarantee the disjunction between $\mathscr{E}$ and $\mathscr{H}$ or int $C \times D$, respectively.

The following Theorem gives sufficient conditions that guarantee that the hypotheses of Proposition 5.1 are fulfilled. 
Theorem 5.1. Suppose that $\mathscr{E}$ and $\mathscr{H}$ admit a linear separation and that int $D \neq \emptyset$.

(i) Let $C=\mathbf{R}_{+}^{\ell}$. Assume that, for every $i=1, \ldots, \ell$, the following system is possible:

$$
f_{i^{-}}(y)-f_{i^{-}}(x)>0, \quad g(x) \in \text { int } D, \quad x \in X,
$$

where, for any $z \in \mathbf{R}^{n}, z_{i^{-}}$denotes the vector $z$ without the $i$-th component. Then, in (5.1) we can suppose $\theta^{*}>0$.

(ii) If there exists $\bar{x} \in X$ such that $g(\bar{x}) \in$ int $D$, then, in (5.1), we can suppose $\theta^{*} \neq 0$.

Proof. (i) Suppose that, $\exists i \in\{1, \ldots, \ell\}$ such that $\theta_{i}^{*}=0$. Then $\left(\theta_{i^{-}}^{*}, \lambda^{*}\right) \neq 0$. Since $S_{i}(y)$ is possible, we find that there exists $\bar{x} \in X$ such that

$$
0<\left\langle\theta_{i^{-}}^{*}, f_{i^{-}}(y)-f_{i^{-}}(\bar{x})\right\rangle+\left\langle\lambda^{*}, g(\bar{y})\right\rangle \leq-\left\langle\theta_{i}^{*}, f_{i}(y)-f_{i}(\bar{x})\right\rangle=0,
$$

which is absurd.

(ii) Suppose that $\theta^{*}=0$ in (5.1). Then, $\lambda^{*} \neq 0$. Since $g(\bar{x}) \in$ int $D$, we have

$$
0<\left\langle\lambda^{*}, g(x)\right\rangle \leq 0
$$

which is absurd. This completes the proof.

Theorem 5.2. $\mathscr{E}$ and $\mathscr{H}$ admit a separating hyperplane of equation

$$
\left\langle\theta^{*}, u\right\rangle+\left\langle\lambda^{*}, v\right\rangle=0, \quad \text { with } \theta^{*} \in \operatorname{int} C^{*}, \lambda^{*} \in D^{*}
$$

iff

$$
\text { cl cone conv } \mathscr{E} \cap \mathscr{H}_{u}=\emptyset \text {. }
$$

Proof. Taking into account Proposition 4.2, it immediately follows from Theorem 3.1 of [5]. This completes the proof.

We recall the following result [10].

Proposition 5.2. Let $B$ be a convex set in $\mathbf{R}^{m}$ and $x \in \operatorname{rbd}(B)$. Then $B$ admits a supporting hyperplane at $x$ and its normal vector belongs to aff $(B-x)$.

Proof. See Lemma 4.2.1 and Remark 4.2.2 of Chapter III in [10].

The following Theorem provides a generalization of the classic Slater constraint qualification.

Theorem 5.3. Suppose that $\mathscr{E}$ and $\mathscr{H}$ admit a proper linear separation and, furthermore,

$$
0 \in \operatorname{ri}(\operatorname{conv}(g(X)-D)) .
$$

Then there exist $\theta^{*} \in C^{*} \backslash\{0\}$ and $\lambda^{*} \in D^{*}$ such that

$$
\left\langle\theta^{*}, u\right\rangle+\left\langle\lambda^{*}, v\right\rangle \leq 0, \quad \forall(u, v) \in \mathscr{E} .
$$

Proof. By Theorem 4.2, the proper linear separation is equivalent to

$$
0 \notin \operatorname{ri}(\operatorname{conv} \mathscr{E}) \text {. }
$$

Since $0 \in \mathscr{E}$, we have $0 \in \operatorname{rbd}(\operatorname{conv} \mathscr{E})$. Applying Proposition 5.2, we obtain that there exist $\left(\theta^{*}, \lambda^{*}\right) \in \operatorname{aff}(\operatorname{conv} \mathscr{E})$ such that (5.4) holds. Since $0 \in \mathscr{E}$, we have $\lambda^{*} \in \operatorname{lin}(\operatorname{conv} \mathscr{E})$. Suppose that $\theta^{*}=0$. Then

$$
\lambda \in \operatorname{lin}(\operatorname{conv}(g(X)-D))
$$


and (5.4) implies

$$
\langle\lambda, v\rangle \leq 0, \quad \forall v \in \operatorname{conv}(g(X)-D)
$$

By (5.3), there exists a neighborhood $U$ of $0 \in \mathbf{R}^{\ell} \times \mathbf{R}^{m}$ such that

$$
V:=U \cap \operatorname{lin}(\operatorname{conv}(g(X)-D)) \subseteq \operatorname{conv}(g(X)-D) .
$$

Taking into account (5.5), we obtain that $\gamma \lambda^{*} \in V$ for $|\gamma|<\varepsilon$, sufficiently small. Since $V \subset$ $\operatorname{conv}(g(X)-D)$, by (5.6), we have

$$
\gamma\left\langle\lambda^{*}, \lambda^{*}\right\rangle \leq 0, \quad \forall \gamma:|\gamma|<\varepsilon
$$

which is impossible for $\lambda^{*} \neq 0$. This completes the proof.

Remark 5.1. If int $D \neq \emptyset$, then (5.3) collapses to the classic Slater condition

$$
\exists \bar{x} \in X \quad \text { s.t. } \quad g(\bar{x}) \in \text { int D. }
$$

\section{SUFFICIENT OPTIMALITY CONDITIONS}

We have seen, in Section 4, that linear separation is equivalent to a saddle point condition for the Lagrangian function $L$. The saddle point condition guarantees that $y$ is a weak v.m.p. if we ensure that the multiplier $\theta^{*}$ is not zero.

Theorem 6.1. Suppose that there exist $\theta^{*} \in C^{*}, \lambda^{*} \in D^{*},\left(\theta^{*}, \lambda^{*}\right) \neq 0$ such that $\left(\lambda^{*}, y\right)$ is a saddle point for $L\left(\theta^{*} ; \lambda, x\right)$ on $D^{*} \times X$. In addition, suppose that there exist $\tilde{x} \in X, \tilde{c} \in C, \tilde{d} \in D$ such that $\left\langle\theta^{*}, f(y)-f(\tilde{x})-\tilde{c}\right\rangle+\left\langle\lambda^{*}, g(\tilde{x})-\tilde{d}\right\rangle<0$ and (5.3) holds. Then $y \in R$ is a weak v.m.p. for $(P)$.

Proof. $\left(\lambda^{*}, y\right)$ is a saddle point for $L\left(\theta^{*} ; \lambda, x\right)$ on $D^{*} \times X$ iff (4.6) and (4.7) are fulfilled.

First, we prove that $y \in R$. Suppose that $g(y) \notin D=\left(D^{*}\right)^{*}$. Then there exits $\bar{\lambda} \in D^{*}$ such that $\langle\bar{\lambda}, g(y)\rangle<0$. Since $D^{*}$ is a cone, we have $\alpha \bar{\lambda} \in D^{*}, \forall \alpha \geq 0$ and

$$
-\alpha\langle\bar{\lambda}, g(y)\rangle \longrightarrow+\infty, \text { for } \alpha \longrightarrow+\infty,
$$

which contradicts (4.6). Therefore $y \in R$. By Proposition 4.2, it follows that the sets $\mathscr{E}$ and $\mathscr{H}$ admit a linear separation and $\left\langle\theta^{*}, u\right\rangle+\left\langle\lambda^{*}, v\right\rangle=0$ is the equation of the separating hyperplane. Let us show that the separation is proper. Suppose that the separation is not proper. Then

$$
\left\langle\theta^{*}, u\right\rangle+\left\langle\lambda^{*}, v\right\rangle=0, \quad \forall(u, v) \in \mathscr{E} \cup \operatorname{cl} \mathscr{H} .
$$

In particular, since $(f(y)-f(\tilde{x}), g(\tilde{x})) \in \mathscr{E}$ and $(\tilde{c}, \tilde{d}) \in c l \mathscr{H}$, we have

$$
\left\langle\theta^{*}, f(y)-f(\tilde{x})\right\rangle+\left\langle\lambda^{*}, g(\tilde{x})\right\rangle=0, \quad\left\langle\theta^{*},-\tilde{c}\right\rangle+\left\langle\lambda^{*},-\tilde{d}\right\rangle=0 .
$$

Summing the previous equalities, we obtain $\left\langle\theta^{*}, f(y)-f(\tilde{x})-\tilde{c}\right\rangle+\left\langle\lambda^{*}, g(\tilde{x})-\tilde{d}\right\rangle=0$, which contradicts the assumptions. Theorem 5.3 guarantees that (5.4) holds with $\theta^{*} \neq 0$. By Proposition 5.1 (ii), we have that $\mathscr{E} \cap($ int $C \times D)=\emptyset$ and Proposition 2.2 yields that $y$ is a weak v.m.p. for $(P)$. This completes the proof.

Remark 6.1. Similarly to Theorem 6.1 , it can be proved that if there exist $\theta^{*} \in \operatorname{int} C^{*}$ and $\lambda^{*} \in D^{*}$ such that $\left(\lambda^{*}, y\right)$ is a saddle point for $L\left(\theta^{*} ; \lambda, x\right)$ on $D^{*} \times X$, then $y$ is a v.m.p. for (P). 
The existence of a saddle point for $L$ is a necessary optimality condition when linear separation holds. To this aim, it is enough to guarantee that $\mathscr{E}$ is a convex set, or, more generally, that cone $\mathscr{E}$ is convex. Suitable generalized convexity assumptions on the function $F(x):=(f(x), g(x))$ turn out to be equivalent to the convexity of the extended image $\mathscr{E}$ or of cone $\mathscr{E}$.

Definition 6.1. Let $V$ be a real topological linear space, $\mathscr{A} \subseteq V$ a convex set, $X$ a nonempty set and $\Phi: X \rightarrow V$ a mapping.

(i) $\Phi$ is said to be $\mathscr{A}$-convexlike on $X$ iff

$$
(1-\alpha) \Phi(X)+\alpha \Phi(X) \subseteq \Phi(X)+\mathscr{A}, \quad \forall \alpha \in(0,1)
$$

(ii) $\Phi$ is said to be $\mathscr{A}$-preconvexlike iff, $\forall \alpha \in[0,1]$, there exists $\rho>0$ such that

$$
(1-\alpha) \Phi(X)+\alpha \Phi(X) \subseteq \rho \Phi(X)+\mathscr{A} .
$$

Definition (i) was introduced in [22] while (ii) was in [25]. If $\mathscr{A}$ is a convex cone and $X$ is a convex set, then it is easy to see that if $\Phi$ is $\mathscr{A}$-convex on $X$, then it is $\mathscr{A}$-convexlike on $X$.

In particular, it is possible to prove the following result $[12,14]$.

Proposition 6.1. $\mathscr{E}$ is convex iff $F$ is $C \times(-D)$-convexlike; (ii) cone $\mathscr{E}$ is convex iff $(f-f(y), g)$ is $C \times(-D)$-preconvexlike.

\section{NECESSARY OPTIMALITY CONDITIONS}

In this section, it will be shown that the separation approach in the image space can be considered as a source for deriving necessary optimality conditions for (P). Following the line developed in [8] for the scalar case, necessary conditions can be obtained by separating the set $\mathscr{H}$ from a suitable approximation of the image $\mathscr{K}$ obtained by assuming $G$-semidifferentiability of the involved function that we briefly recall. The concept of $G$-semidifferentiability of a function was introduced by Giannessi [8] in order to treat in an axiomatic way the theory of generalized directional derivatives. Let $X=\mathbf{R}^{n}$. Denote by $G$ a given subset of the set, say $\mathscr{G}$, of positively homogeneous functions of degree one on $X$, by $\mathscr{C} \subseteq \mathscr{G}$ the set of convex positively homogeneous functions, by $\mathscr{L}$ the set of linear functions.

Definition 7.1. [8] A function $\phi: X \rightarrow \mathbf{R}$ is said lower G-semidifferentiable at $y \in X$ iff there exist functions $\underline{\mathscr{D}}_{G} \phi: X \times X \rightarrow \mathbf{R}$ and $\varepsilon_{\phi}: X \times X \rightarrow \mathbf{R}$ such that

(i) $\underline{\mathscr{D}}_{G} \phi(y ; \cdot) \in G$;

(ii) $\phi(x)-\phi(y)=\underline{\mathscr{D}}_{G} \phi(y ; x-y)+\varepsilon_{\phi}(y ; x-y), \quad \forall x \in X$;

(iii) $\liminf _{x \rightarrow y} \frac{\varepsilon_{\phi}(y ; x-y)}{\|x-y\|} \geq 0$;

(iv) for every pair $(h, \varepsilon)$ of functions which satisfy (i)-(iii), we have epi $h \supseteq$ epi $\underline{\mathscr{D}}_{G} \phi$.

$\underline{D}_{G} \phi\left(y ; \frac{x-y}{\|x-y\|}\right)$ is called the lower $G$-semiderivative of $\phi$ at $y$.

Analogously, a function $\phi: X \rightarrow \mathbf{R}$ is said upper $G$-semidifferentiable at $y \in X$ iff there exist functions $\overline{\mathscr{D}}_{G} \phi: X \times X \rightarrow \mathbf{R}$ and $\varepsilon_{\phi}: X \times X \rightarrow \mathbf{R}$ such that

(i') $\overline{\mathscr{D}}_{G} \phi(y ; \cdot) \in G$;

(ii') $\phi(x)-\phi(y)=\overline{\mathscr{D}}_{G} \phi(y ; x-y)+\varepsilon_{\phi}(y ; x-y), \quad \forall x \in X$; 
(iiii') $\limsup _{x \rightarrow y} \frac{\varepsilon_{\phi}(y ; x-y)}{\|x-y\|} \leq 0$;

(iv') for every pair $(h, \varepsilon)$ of functions which satisfy (i')-(iii'), we have epi $h \subseteq$ epi $\overline{\mathscr{D}}_{G} \phi$.

$\overline{\mathscr{D}}_{G} \phi\left(y ; \frac{x-y}{\|x-y\|}\right)$ is called the upper G-semiderivative of $\phi$ at $\mathrm{y}$.

A function which is both lower and upper $G$-semidifferentiable is called a $G$-differentiable function.

Remark 7.1. We observe that the set of $\mathscr{L}$-differentiable functions coincides with the set of differentiable functions in the classic sense.

Definition 7.2. Let $G \subseteq \mathscr{C}$. The generalized subdifferential of a lower (or upper) $G$-semidifferentiable function $\phi$ at $y$, denoted by $\partial_{G} \phi(y)$, is defined as the subdifferential (see [20]) at $y$ of the convex function $\underline{\mathscr{D}}_{G} \phi(y ; x-y)$ (or $\overline{\mathscr{D}}_{G} \phi(y ; x-y)$ ), that is,

$$
\partial_{G} \phi(y)=\partial \underline{\mathscr{D}}_{G} \phi(y, 0) \quad\left(\text { or } \partial_{G} \phi(y)=\partial \overline{\mathscr{D}}_{G} \phi(y, 0)\right) .
$$

Remark 7.2. If $G \subseteq(-\mathscr{C})$, then the generalized superdifferential of a lower (or upper) $G$ semidifferentiable function $\phi$ is defined as the superdifferential of its concave approximation $\underline{\mathscr{D}}_{G} \phi$ (or $\left.\overline{\mathscr{D}}_{G} \phi\right)$.

In [8], it was shown that, by suitably choosing the set $G$, the upper $G$-semiderivative of the function $\phi$ collapses to the Clarke generalized directional derivative [3].

We next recall the main properties of $G$-semidifferentiable functions.

Proposition 7.1. [17] Suppose that G satisfies the following condition:

$$
\psi_{1}, \psi_{2} \in G \quad \text { implies } \psi_{1}+\psi_{2} \in G .
$$

If $\phi_{1}, \phi_{2}$ and $\phi_{1}+\phi_{2}$ are lower (upper) G-semidifferentiable at $y$, then

$$
\underline{\mathscr{D}}_{G} \phi_{1}+\underline{\mathscr{D}}_{G} \phi_{2} \geq \underline{\mathscr{D}}_{G}\left(\phi_{1}+\phi_{2}\right) \quad\left(\overline{\mathscr{D}}_{G} \phi_{1}+\overline{\mathscr{D}}_{G} \phi_{2} \leq \overline{\mathscr{D}}_{G}\left(\phi_{1}+\phi_{2}\right)\right) .
$$

Proposition 7.2. [17] Suppose that $G$ satisfies the following condition:

$$
\psi \in G \quad \text { implies } \quad \alpha \psi \in G, \quad \forall \alpha>0 .
$$

If $\phi$ is lower (upper) G-semidifferentiable at $y$, then $\forall \alpha>0, \alpha \phi$ is lower (upper) G-semidifferentiable at $y$ with

$$
\alpha \underline{\mathscr{D}}_{G} \phi(y ; x-y) \quad\left(\alpha \overline{\mathscr{D}}_{G} \phi(y ; x-y)\right)
$$

as lower (upper) G-semiderivative.

Let us turn our attention to problem (P), where we assume $X=\mathbf{R}^{n}, C=\mathbf{R}_{+}^{\ell}$ and $D=\mathbf{R}_{+}^{m}$.

Let us introduce the index sets $J:=\{1, \ldots, m\}$ and $J^{0}(y):=\left\{i \in J: g_{i}(y)=0, \varepsilon_{g_{i}}(y ; x-y) \not \equiv\right.$ $0\}$. The following lemma is a generalization of the Linearization Lemma of Abadie [1].

Lemma 7.1. [9] Let the functions $f_{i}, i \in \mathscr{I}$, be upper $\Phi$-semidifferentiable at $y$ and $g_{i}, i \in J$, lower $\Gamma$-semidifferentiable at $y$, where $\Phi, \Gamma \subseteq \mathscr{G}$. If $y$ is a v.m.p. of $(P)$, then the system (in the unknown $x)$ :

$$
\left\{\begin{array}{l}
\overline{\mathscr{D}}_{\Phi} f_{i}(y ; x-y)<0, \quad i \in \mathscr{I}, \\
\underline{\mathscr{D}}_{\Gamma} g_{i}(y ; x-y)>0, \quad i \in J^{0}(y), \\
g_{i}(y)+\underline{\mathscr{D}}_{\Gamma} g_{i}(y ; x-y) \geq 0, \quad i \in J \backslash J^{0}(y), \\
x \in X
\end{array}\right.
$$


is impossible.

The impossibility of system (7.1) is a first order optimality condition for (P), which can be expressed by means of separation arguments in terms of suitable multipliers associated with the $G$-semiderivatives of the objective and the constraint functions.

Theorem 7.1. [9] Let $f_{i}, i \in \mathscr{I}$ be upper $\Phi$-semidifferentiable at $y$, with $\Phi \subseteq \mathscr{C}$ and $g_{i}, i \in J$ be lower $\Gamma$-semidifferentiable at $y$ with $\Gamma \subseteq(-\mathscr{C})$. If $y$ is a v.m.p. of $(P)$, then there exists a vector $(\theta, \lambda) \in \mathbf{R}_{+}^{\ell} \times \mathbf{R}_{+}^{m}$ with $(\theta, \lambda) \neq 0$ such that

$$
\begin{gathered}
\sum_{i \in \mathscr{I}} \theta_{i} \overline{\mathscr{D}}_{\Phi} f_{i}(y ; x-y)-\sum_{i \in J} \lambda_{i} \underline{\mathscr{D}}_{\Gamma} g_{i}(y ; x-y) \geq 0, \in X ; \\
\sum_{i \in J} \lambda_{i} g_{i}(y)=0 .
\end{gathered}
$$

Theorem 7.2. Let $\Phi \subseteq \mathscr{C}$ and $\Gamma=-\Phi$. Suppose that $f_{i} i \in \mathscr{I}$ are upper $\Phi$-semidifferentiable functions, $g_{i} i \in J$ are lower $\Gamma$-emidifferentiable functions at $y \in X$ and the function $\langle\theta, f\rangle-$ $\langle\lambda, g\rangle$ is upper $\Phi$-semidifferentiable at $y$ for any $(\theta, \lambda) \in \mathbf{R}_{+}^{\ell} \times \mathbf{R}_{+}^{m}$. If $y$ is a v.m.p. of $(P)$, then there exists $(\theta, \lambda) \in \mathbf{R}_{+}^{\ell} \times \mathbf{R}_{+}^{m}$, with $(\theta, \lambda) \neq 0$, such that

$$
\left\{\begin{array}{l}
0 \in \partial_{\Phi} L(\theta ; \lambda, y),\langle\lambda, g(y)\rangle=0 \\
\lambda \geq 0, \theta \geq 0, g(y) \geq 0, y \in X .
\end{array}\right.
$$

Proof. Taking into account that $-\underline{\mathscr{D}}_{\Gamma} g=\overline{\mathscr{D}}_{\Phi}(-g)$, the following inequalities hold:

$$
\overline{\mathscr{D}}_{\Phi}(\langle\theta, f\rangle-\langle\lambda, g\rangle)(y ; x-y) \geq\left\langle\theta, \overline{\mathscr{D}}_{\Phi} f(y ; x-y)\right\rangle+\left\langle\lambda, \overline{\mathscr{D}}_{\Phi}(-g)(y ; x-y)\right\rangle \geq 0, \quad \forall x \in X .
$$

The first inequality follows from Proposition 7.1, while the second follows from Theorem 7.1. The previous relation implies that $0 \in \partial_{\Phi} L(\theta ; \lambda, y)$. From Theorem 7.1 (ii), we have the complementarity relation $\langle\lambda, g(y)\rangle=0$. The proof is complete.

The system (7.2) is a generalization to nondifferentiable vector optimization problems of the well-known John conditions for scalar extremum problems. The present scheme allows us to derive the classic first order optimality conditions in the differentiable case.

Theorem 7.3. Let $f_{i}, i \in \mathscr{I}$, and $g_{i}, i \in J$ be differentiable functions at $y$. If $y$ is a v.m.p. of (P), then there exists $(\theta, \lambda) \in \mathbf{R}_{+}^{\ell} \times \mathbf{R}_{+}^{m}$, with $(\theta, \lambda) \neq 0$ such that

$$
\left\{\begin{array}{l}
\sum_{i \in \mathscr{I}} \theta_{i} \nabla f_{i}(y)-\sum_{i \in J} \lambda_{i} \nabla g_{i}(y)=0,\langle\lambda, g(y)\rangle=0, \\
\theta \geq 0, \lambda \geq 0, g(y) \geq 0, y \in X .
\end{array}\right.
$$

Proof. Let $G$ be the set of the linear functions. With $\Phi=\Gamma=G$, we have that the hypotheses of Theorem 7.1 are fulfilled, and it was known [8] that

$$
\begin{aligned}
& \overline{\mathscr{D}}_{G} f_{i}(y ; x-y)=\left\langle\nabla f_{i}(y), \frac{x-y}{\|x-y\|}\right\rangle, i \in \mathscr{I} \\
& \underline{\mathscr{D}}_{G} g_{i}(y ; x-y)=\left\langle\nabla g_{i}(y), \frac{x-y}{\|x-y\|}\right\rangle, i \in J .
\end{aligned}
$$


Therefore, there exists $(\theta, \lambda) \in \mathbf{R}_{+}^{\ell} \times \mathbf{R}_{+}^{m}$ with $(\theta, \lambda) \neq 0$ such that

$$
\sum_{i \in \mathscr{I}} \theta_{i}\left\langle\nabla f_{i}(y), \frac{x-y}{\|x-y\|}\right\rangle-\sum_{i \in J} \lambda_{i}\left\langle\nabla g_{i}(y), \frac{x-y}{\|x-y\|}\right\rangle \geq 0, \quad \forall x \in X
$$

. The previous condition is equivalent to

$$
\left\langle\sum_{i \in \mathscr{I}} \theta_{i} \nabla f_{i}(y)-\sum_{i \in J} \lambda_{i} \nabla g_{i}(y), z\right\rangle \geq 0, \quad \forall z \in \mathbf{R}^{n} \text { with }\|z\|=1 .
$$

Since $z$ is arbitrary, we obtain:

$$
\sum_{i \in \mathscr{I}} \theta_{i} \nabla f_{i}(y)-\sum_{i \in J} \lambda_{i} \nabla g_{i}(y)=0 .
$$

From (ii) of Theorem 7.1, we have that $\langle\lambda, g(y)\rangle=0$. This completes the proof.

Under suitable regularity assumptions on $(\mathrm{P})$, which ensure that the multipliers $\theta$ are different from the zero vector in $\mathbf{R}^{\ell}$, system (7.2) is a generalization to nondifferentiable Vector Optimization Problems of the classic Kuhn-Tucker conditions.

\section{Acknowledgements}

The first and the second author were partially supported by the project sponsored by University of Pisa under Grant PRA-2017-05. The third author, who is a member of the National Group for Mathematical Analysis, Probability and their Applications (GNAMPA) of the National Institute of Higher Mathematics (INDAM), was partially supported by University of Catania (Piano della Ricerca 2016/2018 Linea di intervento 2).

\section{REFERENCES}

[1] J. Abadie, On the Kuhn-Tucker theorem. Nonlinear Programming, North-Holland, Amsterdam, 1967.

[2] A. Berman, Cones, matrices and mathematical programming, Lecture notes in Economics and Mathematical Systems, Springer-Verlag, Berlin, 1973.

[3] F.H. Clarke, Optimization and Nonsmooth Analysis, John Wiley, New York, 1984.

[4] H.W. Corley, An existence result for maximization with respect to cones, J. Optim. Theory Appl. 31 (1980), 277-281.

[5] F. Flores-Bazan, G. Mastroeni, C. Vera, Proper or weak efficiency via saddle point conditions in cone constrained nonconvex vector optimization problems, J. Optim. Theory Appl. 181 (2019), 787-365.

[6] F. Giannessi, Constrained Optimization and Image Space Analysis, Springer, 2005.

[7] F. Giannessi, Theorems of the alternative and optimality conditions, J. Optim. Theory Appl. 42 (1984), 331365.

[8] F. Giannessi, Semidifferentiable functions and necessary optimality conditions, J. Optim. Theory Appl. 60 (1989), 191-241.

[9] F. Giannessi, G. Mastroeni, L. Pellegrini, On the theory of vector optimization and variational inequalities. Image space analysis and separation. In "Vector Variational Inequalities and Vector Equilibria. Mathematical Theories.”, F. Giannessi, Ed., Kluwer Acad. Publ., Dordrecht, Boston, London pp. 153-215, 2000.

[10] J.B. Hiriart-Urruty, C. Lemarechal, Convex Analysis and Minimization Algorithms, Vol. 1, Springer Verlag, Berlin, Germany, 1993.

[11] R. Hartley, On cone-efficiency, cone-convexity and cone-compactness, SIAM J. Appl. Math. 34 (1978), 211-222.

[12] J. Li, G. Mastroeni, Image convexity of generalized systems with infinite-dimensional image with applications, J. Optim. Theory Appl. 169 (2016), 91-115.

[13] G. Mastroeni, L. Pellegrini, On the image space analysis for vector variational inequalities, J. Ind. Manag. Optim. 1 (2005), 123-132. 
[14] G. Mastroeni, T. Rapcsak, On convex generalized systems, J. Optim. Theory Appl. 104 (2000), $605-627$.

[15] G. Mastroeni, M. Pappalardo, On the existence of solutions to vector optimization problems. In "Equilibrium problems: nonsmooth optimization and variational inequality methods", Nonconvex Optim. Appl. 58, Kluwer Acad. Publ., Dordrecht, pp. 175-185, 2001.

[16] M. Pappalardo, Image space approach to penalty methods, J. Optim. Theory Appl. 64 (1990), 141-152.

[17] M. Pappalardo, On semidifferentiable functions, Optimization 24 (1992), 207-217.

[18] F. Raciti, Equilibrium conditions and vector variational inequalities: a complex relation, J. Glob. Optim. 40 (2008), 353-360.

[19] A. Raith, M. Ehrgott, On vector equilibria, vector optimization and vector variational inequalities, J. MultiCriteria Decision Anal. 18 (2011), 39-54.

[20] R.T. Rockafellar, Convex Analysis, Princeton University Press, Princeton, 1970.

[21] Y. Sawaragi, H. Nakayama, T. Tanino, Theory of multiobjective Optimization, Academic Press, New York, 1985.

[22] F. Tardella, On the image of a constrained extremum problem and some applications to the existence of a minimum, J. Optim. Theory Appl. 60 (1989), 93-104.

[23] F. Tardella, Some topological properties in optimization theory, J. Optim. Theory Appl. 60 (1989), $105-116$.

[24] D.H. Wagner, Semicompactness with respect to a Euclidean cone, Canadian J. Math. 29 (1977), 29-36.

[25] R. Zeng, R.J. Caron, Generalized Motzkin alternative theorems and vector optimization problems, J. Optim. Theory Appl. 131 (2006), 281-299. 